\title{
The Ice Particle and Aggregate Simulator (IPAS). Part I: Extracting Dimensional Properties of Ice-Ice Aggregates for Microphysical Parameterization
}

\author{
VANESSA M. PrZybylo AND Kara J. Sulia \\ Atmospheric Sciences Research Center, University at Albany, State University of New York, \\ Albany, New York \\ CARL G. SCHMITT \\ National Center for Atmospheric Research, Boulder, Colorado \\ ZACHARY J. LEBO \\ University of Wyoming, Laramie, Wyoming \\ WILLIAM C. MAY \\ Atmospheric Sciences Research Center, University at Albany, State University of New York, \\ Albany, New York
}

(Manuscript received 6 July 2018, in final form 27 February 2019)

\begin{abstract}
Aggregation, the process by which two or more ice particles attach to each other, is typically observed in clouds that span a range of temperatures and is influenced by the crystal shape (habit). In this study, the resulting characteristics of ice-ice two-monomer aggregation is investigated, which is expected to improve microphysical parameterizations through more precise aggregate characteristics and in turn better predict the rate of aggregation and snow development. A systematic way to determine the aspect ratio of the aggregate was developed, which takes into account the expected falling orientations, overlap of each monomer, and any contact angle that may form through so-called constrained randomization. Distributions were used to obtain the most frequent aspect ratio, major axis, and minor axis of aggregated particles with respect to the monomer aspect ratio. Simulations were completed using the Ice Particle and Aggregate Simulator (IPAS), a model that uses predefined three-dimensional geometries, (e.g., hexagonal prisms) to simulate ice crystal aggregation and allows for variation in crystal size, shape, number, and falling orientation. In this study, after collection in a theoretical grid space, detailed information is extracted from the particles to determine the properties of aggregates. It was found that almost all monomer aspect ratios aggregate to less extreme aggregate aspect ratios at nearly the same rate. Newly formed aggregate properties are amenable to implementation into more sophisticated bulk microphysical models designed to predict and evolve particle properties, which is crucial in realistically evolving cloud ice mass distribution and for representing the collection process.
\end{abstract}

\section{Introduction}

Aggregation is the process by which two or more atmospheric ice particles combine. The ice particles can be individual grown crystals or aggregates and are typically observed in clouds that span a range of temperatures,

Corresponding author: Vanessa M. Przybylo, vprzybylo@ albany.edu and are influenced by the crystal shape (habit). Particles that fall through different temperature regimes acquire distinct growth characteristics through vapor deposition that vary their habit and fall speed (Lamb and Verlinde 2011; Connolly et al. 2012; Phillips et al. 2015). Individual crystals (monomers) and aggregates grow in mass and size through vapor deposition in environments supersaturated with respect to ice via the Wegener-Bergeron-Findeisen (WBF) process. Situations 
arise where the environment is supersaturated with respect to ice, yet subsaturated with respect to liquid, where depositional growth can lead to rapid glaciation (Pruppacher and Klett 1997; Korolev and Isaac 2003; Korolev 2007). As ice crystals grow and continuously uptake vapor, the vapor pressure continues to drop. In subsaturated conditions with respect to liquid, cloud droplets will evaporate acting to replenish vapor, which further aids the maintenance of the WBF process. Regardless of the ice growth process, increases in terminal velocities of monomers and aggregates can lead to subsequent collection of smaller particles by larger ones, enhancing fallout and sedimentation rates (Hobbs and Scott 1965; Connolly et al. 2005). It is critical to parameterize aggregate sedimentation accurately as the inherent size and mass of aggregates quickly influence the overall cloud-phase partitioning and lifetime.

The formation of snow through vapor growth and aggregation is an important component to the development of precipitation. Slowly evolving midlatitude systems with high ice water content and long particle resident times are especially conducive to hydrometeor growth and increased sedimentation rates. For example, aboveexpected rain and snowfall amounts detected at the surface underneath the stratiform region of midlatitude squall lines examined by Rutledge and Houze (1987) and Biggerstaff and Houze (1991) were resolved by a front-to-rear current that saturated the environment for enhanced vapor deposition onto existing ice nuclei. After nucleation took place and saturation diminished, ice-ice aggregation, deemed snow for the purposes of this study, dominated the growth process and provided for the primary source of stratiform rain upon falling through the melting layer toward the surface. Moreover, Cotton et al. (1986) found that orographic effects also expedited ice crystal growth and cloud liquid water depletion, which redistributed surface precipitation in simulated orographic flow over northern Colorado.

The advection of hydrometeors through regions with varying saturation and temperatures further reconfigures the water-phase separation in clouds. In such cases, crystal habit is altered through preferred growth of one axis length over the other, which leads to crystals that are spherical (isometric) transforming to more extreme ${ }^{1}$ habits. As particles proceed to grow nonspherically, curvature and vapor density gradients along the edges of the crystals and aggregates are enhanced (Lamb and Hobbs 1971; Chen and Lamb 1994; Sulia and Harrington 2011).

\footnotetext{
1 "Extreme" habits are defined as those that deviate substantially from spherical with aspect ratios $\phi \gg 1$ or $\phi \ll 1$.
}

This change in crystal habit along with falling orientation strongly influences the aggregate collection efficiency, or the likelihood of hydrometeors to collide and "stick" through interlocking dendritic arms, as particles fall from colder to warmer temperatures (Passarelli 1978; Connolly et al. 2012; Thériault et al. 2012; Rogers and Yau 1989; Hosler and Hallgren 1960; Phillips et al. 2015).

Further, the efficiency of collection directly modifies both monomer ice and aggregate concentrations, subsequently altering the precipitation produced in a given system due to the increase in fall speed with increasing aggregation rates. For instance, numerical experiments and several flight observations in stratified tropical cyclone anvils and mesoscale convective systems have shown that high concentrations of aggregates contributed to the precipitation within the trailing stratiform region behind the leading convection (Jorgensen 1984; Biggerstaff and Houze 1991). Additionally, Churchill and Houze (1984) confirm that growth by vapor deposition and successive aggregation during the Winter Monsoon Experiment (1978) occurred within the relatively weak updraft of the stratiform region where nonuniformity in habit was apparent.

From laboratory experiments and observations, aggregation is favorable in clouds that range between $-25^{\circ}$ and $0^{\circ} \mathrm{C}$ at cloud base and is more favorable with higher ice crystal concentrations (Phillips et al. 2015; Cotton et al. 1986); however, aggregation can take place in clouds with temperatures as low as $-60^{\circ} \mathrm{C}$ (Connolly et al. 2005). Depending on the temperature regime, crystal fall speeds are varied through changes in area, mass, drag coefficients, riming, surface sticking efficiencies, etc. For example, crystals in the dendritic growth zone around $-15^{\circ} \mathrm{C}$ fall slowly, have longer interactions with neighboring crystals, and are more effective at collecting due to interlocking branches. In this case, sintering, or the growth from vapor at the point of contact, enhances the collection efficiency through lengthy interactions and a bond that can form during collision (Connolly et al. 2012). Also, crystal surfaces that have higher molecular energy (warmer temperatures) may exhibit a "quasi-liquid layer" that supplements aggregation efficiencies (Kuroda and Lacmann 1982). Even though a liquid layer may be present on crystal surfaces, it has been proven that the interlocking of branches (e.g., the dendritic growth zone) is the governing factor in determining sticking efficiencies (Connolly et al. 2012).

The work presented herein explores aggregate properties to better elucidate characteristics of aggregation in detail. This investigation provides the necessary stepping stone between simulating aggregation and effectively 
representing this complex process in numerical models. A background on past aggregation schemes and laboratory experiments follows.

\section{Background}

The key to representing aggregation in numerical models is to understand the complexities associated with the process and determine the best method to parameterize aggregate characteristics. To that end, laboratory investigations are typically used to derive characteristics such as aggregation efficiency and mass increase rates due to aggregation. Hosler and Hallgren (1960) used a single spherical ice target (127-360 $\mu \mathrm{m}$ in diameter) subject to an airstream of crystals to categorize the number of sticking crystals by measuring the increase in particle mass with time as a function of temperature. However, the particles used in the study were small ( $\leq 18 \mu \mathrm{m}$ in diameter), which likely ignored the effects of significant branched growth on the ice crystals and, thus, preferential particle interlocking. This experiment was limited by growth time, external dynamical flow, a limiting number of habits based on temperature, and naturally formed collector particles (crystals or aggregates that are nonspherical), among others, leading to necessary and important additional model simulations such as the present study.

Connolly et al. (2012) used a cloud chamber with two cloud particle imager (CPI) probes that took pictures at the base and halfway up (separated by $3.8 \mathrm{~m}$ ) to study early efficiency rates of aggregation determined using the variation in ice concentration measured by the two CPIs. To interpret the data extracted from these experiments, numerical models were used, particularly to compare mass, concentration, and anticipated habit. While Connolly et al. (2012) appropriately parameterize ice crystal growth via deposition following the methodology of Chen and Lamb (1994), they rely on traditional mass-dimensional $(m-D)$ and area-dimensional relationships for determining complex aggregate characteristics, requiring assumptions of aggregate contact angle and projected area. Such relationships have inherent shortcomings as expressed in Harrington et al. (2013) and Sulia et al. (2013): mainly, the constraint of particle mass, density, and habit changes to the average particle growth histories, which does not account for the nonlinear relationship between vapor diffusion and shape evolution (Sulia et al. 2013).

The same shortcomings of these spherical or $m-D$ relationships for ice vapor growth can be applied to past aggregation models, where particle shape, size, and/or density are not properly evolved (Lin et al. 1983;
Reisner and Rasmussen 1998; Gilmore et al. 2004; Reinhardt and Wacker 2004; Thompson et al. 2008). As with vapor-grown monomers, traditional aggregation models not only disregard the monomer habits that compose the aggregate, but also diagnose aggregate characteristics, such as mass, shape, and density, which do not evolve prognostically. For example, some constant density assumptions (e.g., $100 \mathrm{~kg} \mathrm{~m}^{-3}$ ) are only accurate for snow around $1.5 \mathrm{~mm}$ in diameter but become inaccurate with deviations from this particle size (Thompson et al. 2008).

Aggregation models have been updated to obtain a nonspherical shape with a density that varies inversely with diameter and uses specific habit classifications of Locatelli and Hobbs (1974) for more precise $m-D$ relationships (Reisner and Rasmussen 1998; Woods et al. 2007; Thompson et al. 2008). However, in the bulk microphysical scheme of Woods et al. (2007), the habit-dependent approach with specific classifications from Locatelli and Hobbs (1974) did not improve quantitative precipitation forecasts beyond the much simpler constant-coefficient, $m-D$ relationships of previous models and was more computationally expensive; hence, a simpler relationship from Cox (1988) that assumes consistent fractal-like aggregates was used by Thompson et al. (2008). Previous models also require separate equations for the mixing ratios of snow for different habit types that subsequently feed back into the behavior of the microphysical scheme (Woods et al. 2007).

A new bulk scheme, the predicted particle properties (P3) scheme (Morrison and Milbrandt 2015; Morrison et al. 2015), that evolves particle properties temporally and spatially instead of separating ice into different predefined categories has since been developed, which includes a prognostic equation for aggregates. This model does predict and evolve mean density and hydrometeor size from a multitude of growth mechanisms and is an example of a bulk microphysical model that could benefit from the subsequent work.

In another recent study, Hashino and Tripoli (2011b) developed a habit-dependent aggregation model with the Spectral Ice Habit Prediction System (SHIPS) to investigate the effects of crystal habit on aggregation processes. SHIPS uses $m-D$ relationships to diagnose aggregate characteristics through collisional crosssectional area and terminal velocity for each evolving mass bin that are characterized by a representative habit type (seven were used). Simple diagnoses and categorization of crystals fails to encapsulate the wide range of both monomer and aggregate shapes that can form. Particular drawbacks to bin microphysical schemes are the immense computational expense and lack of particle 
characteristic evolution, both of which should be avoided in microphysical schemes.

Aggregation schemes require sophistication to realistically model growth rates from vapor diffusion and handle the advection of particles into different growth regimes. While progress has recently been made in both modeling and laboratory applications to understand and represent aggregation, deficiencies remain. Laboratory efforts are constrained by their inherent exclusivity and inability to garner robust statistics, which would require an unrealistic number of experiments. Models, on the other hand, are constrained by assumptions required for bulk representation, many of which rely on unrealistic autoconversion thresholds for hydrometeor categorization as stated in Milbrandt and Morrison (2013), Morrison and Milbrandt (2015), and Morrison et al. (2015). To augment these limitations, the Ice Particle and Aggregate Simulator (IPAS; Schmitt and Heymsfield 2010, 2014) is used in this study to demonstrate the influence of monomer habit on aggregation. IPAS can be thought of as a theoretical laboratory that allows for variation in initial conditions (such as aspect ratio of the crystals) and has the capability to vary how the crystals fall and collect. The work presented below exemplifies the intricacies of IPAS and its ability to replicate aggregation and extract detailed aggregate properties not found elsewhere. The usefulness of IPAS can transpire with verification to CPI data in future investigations, and derived properties from IPAS (discussed in detail below) will be used as key components for representing aggregation in microphysical schemes.

\section{IPAS model description}

IPAS is a box model that uses hexagonal prisms to simulate ice crystal aggregation. It simulates columns and plates as the primary habits to be collected, determined by the aspect ratio of randomly oriented 12-point hexagonal prisms. Each model run is initialized with monomer $a$ - and $c$-axis lengths, number of aggregates $n_{a}$ to be created for each aspect ratio $(\phi=c / a)$, and number of monomers $n_{m}$ within each aggregate. These initializations not only allow for control over particle shape and relative size but also allow for statistical analyses over a large dataset of varying aggregate formations. Note that flow dynamics around each crystal is not implemented due to massive computational power that would limit the statistical nature of IPAS. While IPAS is currently able to form aggregates with 2-300 monomers, studies herein only simulate the initial aggregation process created by the collision between two monomers (i.e., $n_{m}=2$ ). IPAS was developed by Schmitt and Heymsfield (2010) using a complex graphical gridded interface originally written in Interactive Data Language (IDL). The original IDL implementation, however, is limited by its reliance on plots to calculate crystal properties, which makes it impractical for larger-scale simulations. To address this limitation, the Extreme Collaboration, Innovation, and Technology (xCITE) laboratory, a software development, data analytics laboratory at the Atmospheric Science Research Center reimplemented the core IPAS functionality in Python and replaced the graphical, plot-based calculations with geometric calculations. Namely, the Python version of IPAS is highly dependent on the "shapely" package, which is used for the manipulation and analysis of geometric objects in the Cartesian plane. As a result, the Python implementation achieved a near 100-time speedup in run time for one aspect ratio with 300 , two-monomer aggregates. Due to the random nature of IPAS, it is not possible to duplicate exact calculations, but both the IDL and Python versions appear to produce statistically identical results; thus, the Python version was used for the purpose of this study.

\section{a. Aggregation design in IPAS}

A "seed" crystal is held steady at an orientation that produces the maximum projected area from overhead, which is determined by reorienting the crystal $n$ times so that the orientation with the maximum projected area is selected. A certain degree of tilt from the face with the major-axis length is expected, where the maximum projected area typically includes "overhang" from the edge of the crystal, producing a slightly larger projected area compared to the face with the major-axis length alone (Fig. 1). A second identical crystal is then randomly placed above the seed crystal, again with its maximum dimension perpendicular to the flow in a three-dimensional domain (Fig. 2). Because the falling crystal is introduced to the domain from above, plates (columns) usually only aggregate from the collision of the basal (prism) faces (Fig. 3). Thicker plates (columns) have a better chance for prism (basal) face aggregation due to increased tilt, whereas isometric hexagonal crystals do not have any preference over which faces collect. Aggregation is assumed if any part of the vertically falling crystal and the collecting seed crystal touch (i.e., gravitational sweep out volume is used), with no bearing on efficiency for coalescence (or sticking). Note that while collection (collision and sticking) efficiency is a key component in aggregation, the current design of IPAS does not address this unknown, but will be considered in future work.

There are three variables that characterize the aggregate geometry directly: 


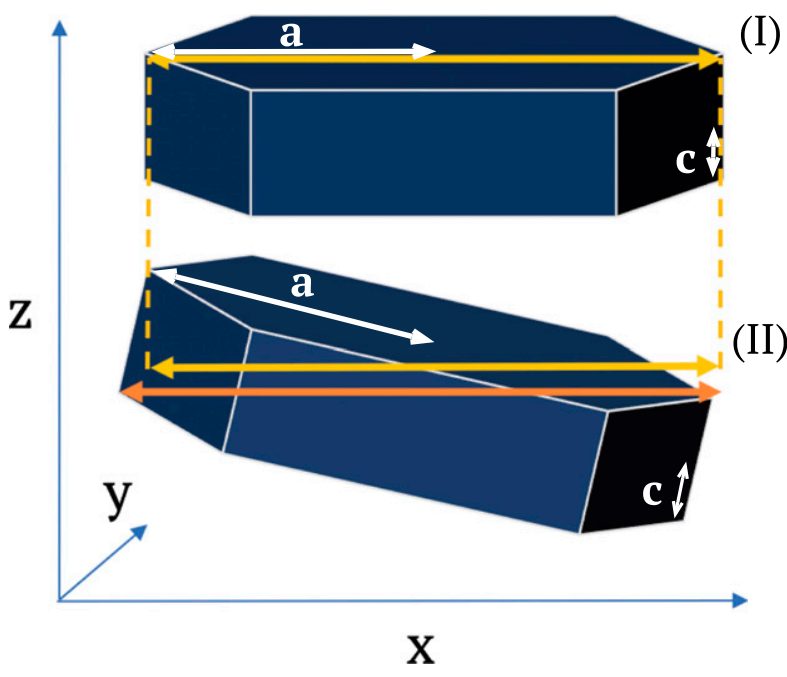

FIG. 1. (top) A hexagonal plate falling with its $a$ axis completely perpendicular to the flow and (bottom) tilting of the hexagonal plate to produce a larger projected dimension/area (orange arrow) as viewed from above. Comparison illustrates that the maximum projected area does not always orient the monomer with its major axis (yellow arrows) perpendicular to the flow.

1) Overlap-The percentage of pixels that both crystals occupy in the $x-y$ plane (Fig. 3, right).

2) Offset-The degree to which one monomer is rotated relative to the other in the $x-y$ plane. For example, two columns stacked parallel have no offset, whereas two columns stacked perpendicular ("X" shape) have a $90^{\circ}$ offset (Fig. 3, right).

3) Contact angle-The degree of rotation measured in either the $x-z$ or $y-z$ plane of one crystal relative to the other (vertical angle; Fig. 3, left).

IPAS allows the crystals to aggregate with any percentage of overlap or degree of offset in the $x-y$ plane; the contact angle is a function of aspect ratio (see below). Higher overlap and smaller contact angles between crystals are suggested to directly relate to increased aggregation efficiency, with overlap taking precedence (Higuchi 1960; Kajikawa and Heymsfield 1989). Intrinsic to the IPAS structure at this stage, every two crystals placed in the domain will collect despite the requirement for varied fall speeds, efficiency values, or the like. A simplistic initial application that involves identical crystals is needed to understand the steps involved in simulated aggregation before moving onto varied crystal sizes and shapes.

From Schmitt et al. (2016), it is shown that on average, single crystals are rarely larger than a few hundred micrometers from CPI data in 10 global datasets analyzed, so in the aggregation of two identically small particles here, turbulent effects and wake capture are

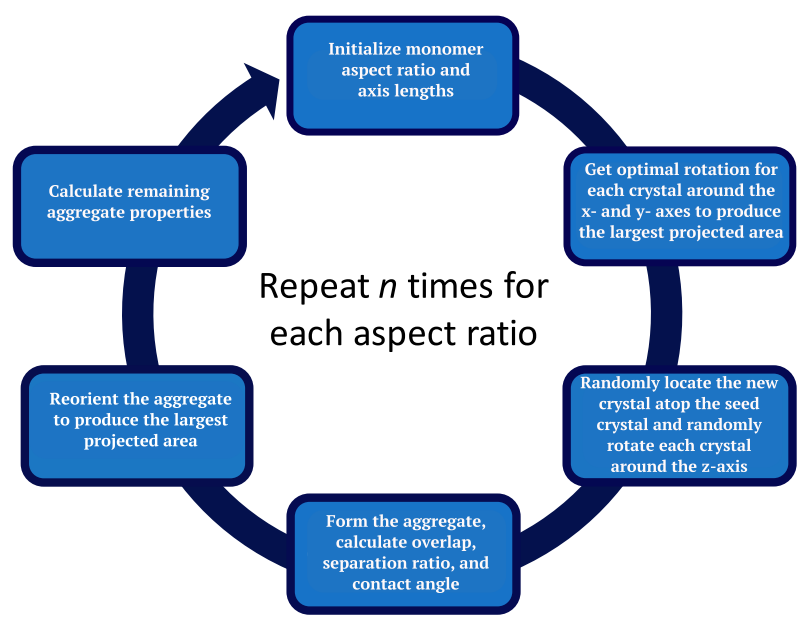

FIG. 2. Sequence of operation in the IPAS framework. This sequence is repeated $n$ times per aspect ratio for aspect ratios typically varying from $0.01 \leq \phi \leq 100$.

likely unimportant given the necessities for such large particles and large differences in terminal velocity. Currently, the predominate motivation to using IPAS is the extraction of aggregate dimensions after aggregation has taken place. The intent is for bulk models to then generate a distribution of aggregate aspect ratios centered around a particular IPAS-generated value, where variability in size and fall speeds naturally results from both depositional growth and continued aggregate formation. Newly formed aggregate properties using IPAS produce a way to avoid empirically setting aggregate characteristics across a range of aspect ratios that never appropriately evolve.

\section{b. Aggregate aspect ratio calculation}

To develop a quasi-continuous representation of ice particles as they advance from single crystal to aggregate, it is necessary to calculate the aspect ratio of the new aggregate. This is done to mimic the $c$ and $a$ axes of the monomer aspect ratio, where $\phi=c / a$. As with the monomer crystals prior to aggregate formation, the resulting aggregate is reoriented $n$ times so that the orientation with the maximum projected area is selected (i.e., the maximum dimension is perpendicular to the assumed flow). The major-axis length was then determined from an ellipse that was fit to the aggregate in the $x-y$ plane following Fanning (2002) (Fig. 3) and used as the aggregate $a_{a}\left(c_{a}\right)$ axis for plates (columns) after reorientation (Fig. 3). Although not a true plate or column particle once aggregated, the resulting aggregate aspect ratio is categorized as "plate-like" or "column-like" based on the habits of the monomers that compose the aggregate. For example, the major dimension of a plate-like aggregate $(a>c)$ will be labeled as $a_{a}$ where $a_{a}>c_{a}$. 

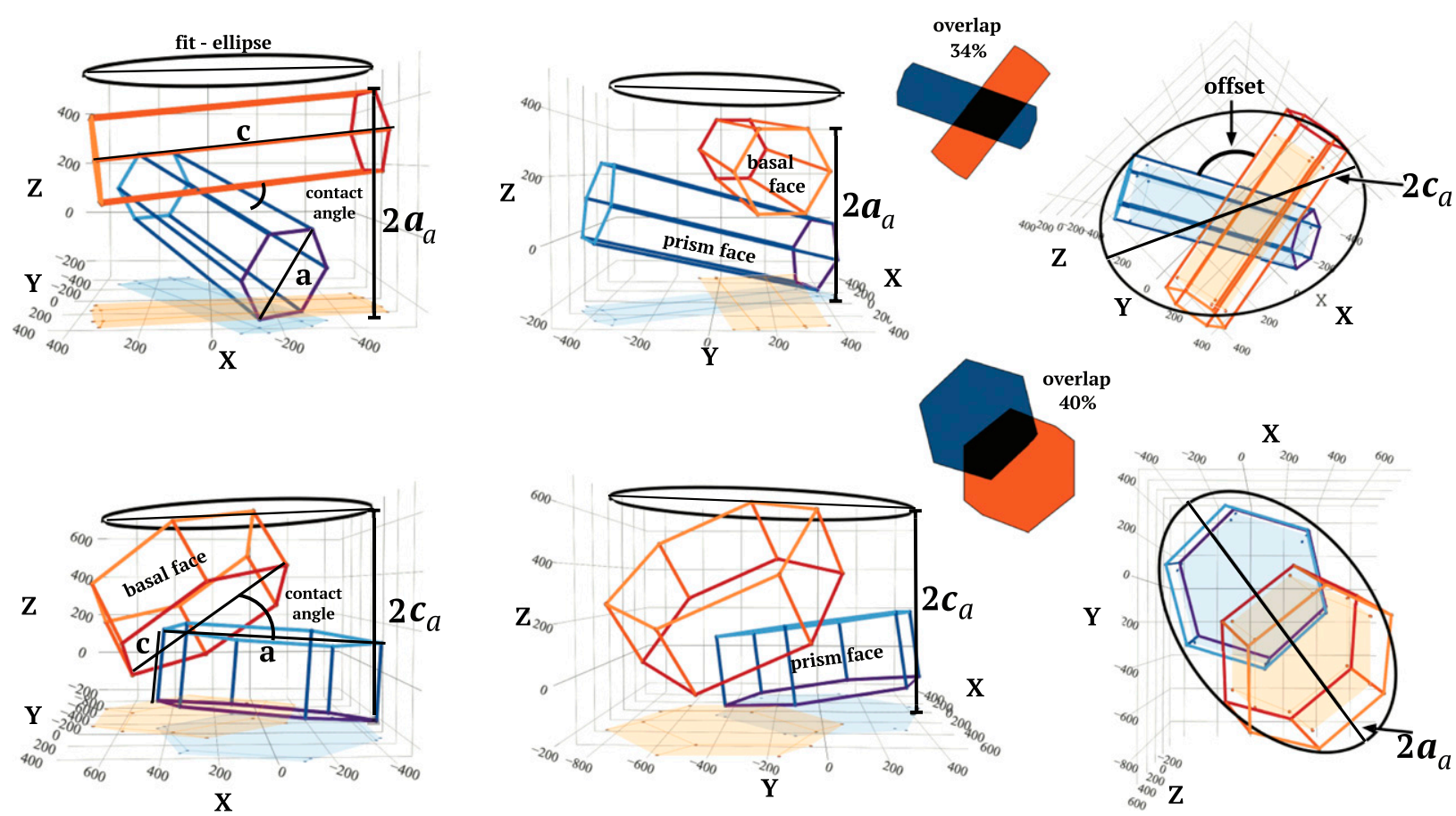

FIG. 3. View of (left) $x-z$, (center) $y-z$, and (right) $x-y$ planes of a two-monomer (top) columnar and (bottom) planar aggregates. Offset in the right panel is defined by rotation in the $x-y$ plane or angle between the two monomers' $c(a)$ axes for columns (plates). Overlap is the ratio of the overlapped area to the total area of the seed crystal (blue). Contact angle in the top-left panel is the angle between the basal (prism) faces of the two planar (columnar) monomers. The new aggregate major-axis length is the maximum dimension of the fit ellipse in the $x-y$ plane in the right panel; the minor-axis length is the depth, or the distance between the maximum and minimum points in the $z$ dimension (see left and middle panels). Shaded projections indicate the crystal projected areas.

The determination of the minor-axis length of the aggregate, $c_{a}\left(a_{a}\right)$ for plates (columns), was tested using two different methods (Fig. 4). In the end, a depth measurement was implemented, defined as the distance between the minimum and maximum points of the aggregate along the $z$ axis. This methodology provides a measurement that is always perpendicular to the major axis retrieved from overhead, spanning the entirety of both monomers, and best represents the minor axis (Fig. 4).

\section{c. IPAS output consistency}

Laboratory studies such as those outlined above that grow and precipitate crystals in chambers including ventilation effects are limited by the lack of reproducibility. However, in the laboratory, wake turbulence and turbulent flow is considered. An advantage to a model such as IPAS is ideal in the ability to perform a multitude of sensitivity tests that can be run with relative consistency.

The number of aggregates per aspect ratio was varied from 100 to 1000 to show that the characteristic aggregate aspect ratio (value of the fitted distribution peak) minimally varies with more data per aspect ratio and does not improve upon the already inherent variability in IPAS (Fig. 5a). While more aggregates led to a smoother distribution, due to lengthy run times, 300 aggregates per aspect ratio were chosen for subsequent

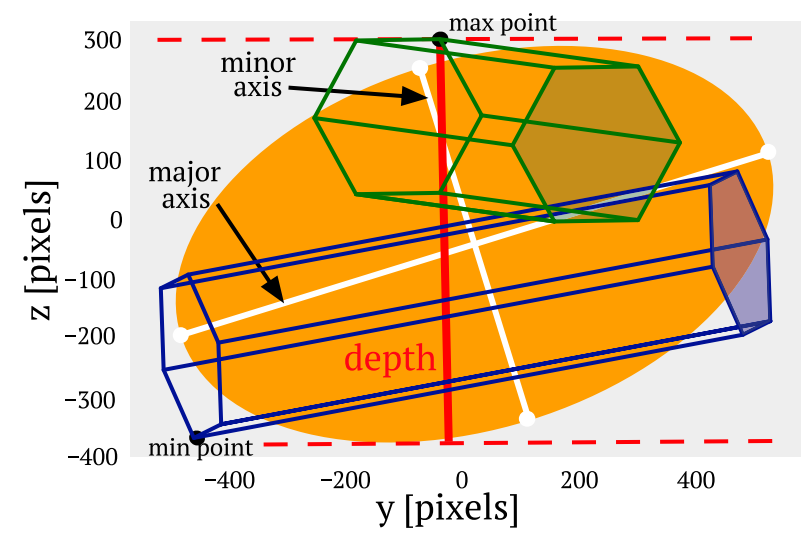

FIG. 4. Schematic of the fit-ellipse algorithm used to determine the minor and major axes (white lines) in the $x$ orientation (note that calculations for the major axis use the $z$ orientation). Black dots represent the maximum and minimum points along the $z$ axis and the red line represents the depth of the aggregate. Both monomers have the same shape and size. 

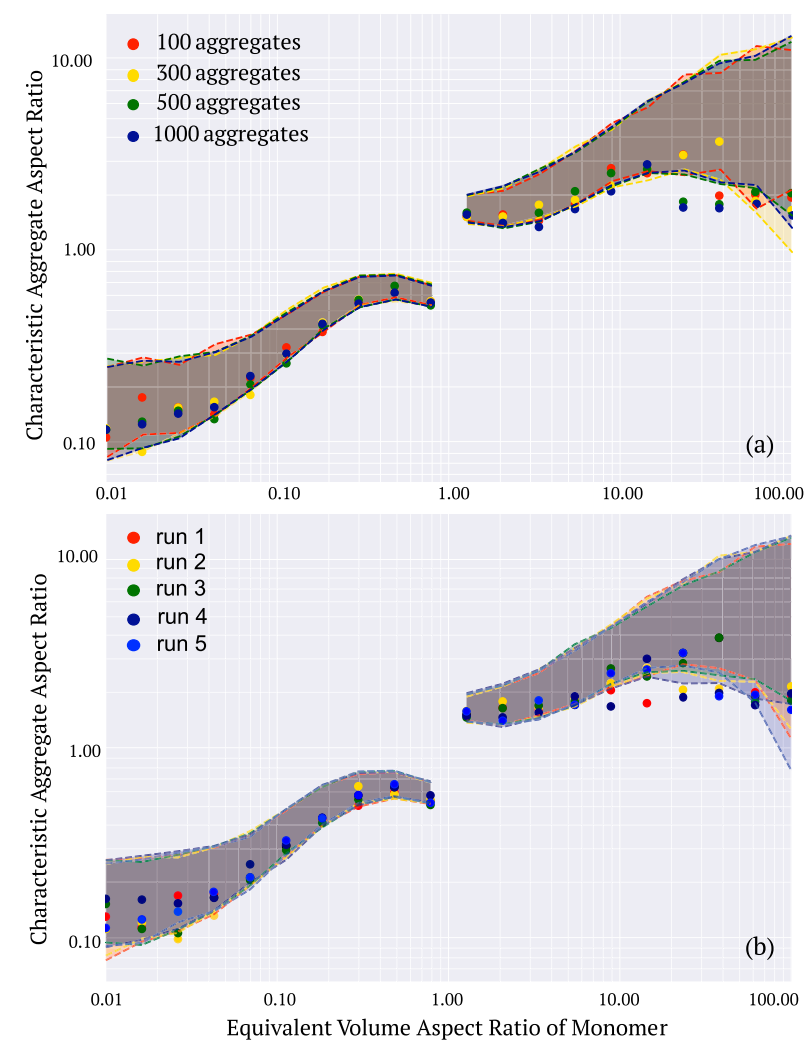

FIG. 5. (a) Characteristic aggregate aspect ratio (colored dots) from a gamma distribution varying the number of aggregates per aspect ratio to show minimal variability between simulations. (b) Multiple simulations of the characteristic aggregate aspect ratio from the best distribution for 300 aggregates per monomer aspect ratio. Shading represents the mean plus and minus one standard deviation. All simulations follow the method that optimizes reorientation of the monomers and only randomizes the placement of the falling crystal over the seed crystal and rotation around the $z$ axis. This is done to show reproducibility between simulations.

simulations. The resulting trend across all monomer aspect ratios remains consistent for such a sample size, which ensures that an appropriate anticipated range and frequency of aggregate aspect ratios can be represented with 300 aggregate formations per aspect ratio and is not compromised for calculation efficiency (Fig. 5a).

Due to the inherent randomization built into the orientation methodology, no two aggregates or distribution thereof will be the same, and so repeated simulations with identical initial conditions will never produce $100 \%$ identical results. While this feature captures the "randomness" found in nature, consistency is still desired even in stochastic models. To show that the aggregate aspect ratio is nearly constant between simulations, five instances with the same initializations were completed (Fig. 5b). While not identical, only slight variations in the simulations appear; the greatest difference occurs for extreme columns and plates since there are more possible aggregate configurations, and thus characteristic values as crystal size is increased (e.g., variability in offset, overlap, and contact angle). Moreover, the obvious similarities between Figs. $5 \mathrm{a}$ and $5 \mathrm{~b}$ indicate that the variability in resulting aggregate distribution size is due to the stochastic nature of IPAS and likely not sensitive to the number of aggregates formed per aspect ratio.

\section{d. IPAS aggregate distributions}

To determine statistically reliable aggregate characteristics, simulations were run to generate 300 aggregates per aspect ratio ranging from 0.01 to 100 logarithmically incremented to produce 50 aspect ratios (or 20 aspect ratios for longer simulations). Each aggregate is composed of two monomers, where each monomer is initialized by an equivalent volume of 1000 pixels or an equivalent spherical volume radius of 10 pixels. The pixel count of each axis is then determined using this volume and desired aspect ratio to ensure consistency of size across all aspect ratios. Equivalent spherical volume radius takes into account both the depth and major-axis dimension and is calculated as $r=\sqrt[3]{a^{2} c}$. The leading coefficient (e.g., $4 \pi / 3$ or $3 \sqrt{3} / 2$ ) is omitted for applicability to both spheroids and hexagonal prisms. The crystals in both the IDL and Python versions are measured in pixels and thus are unitless. Defining monomer attributes as a function of crystal aspect ratio allows for this methodology.

Once the ice-ice aggregate is formed, the aggregate aspect ratio is determined using the method defined in section $3 \mathrm{~b}$. This process is repeated $n=300$ times (i.e., with each formation of a new aggregate), populating a histogram, or binned distribution, of aggregate aspect ratios. All other aggregate properties, including dimensions, density, overlap, and contact angle, are similarly computed with each aggregate formation, and similarly are used to populate a separate binned distribution. Hence, a unique binned distribution is produced for each monomer aspect ratio and for each desired aggregate property.

The intent behind the design of IPAS is so that the derived aggregate parameters are amenable for integration into bulk microphysical models. As such, for every aggregate parameter (aggregate aspect ratio, overlap, contact angle, etc.), a bulk characteristic value is determined. The characteristic value of the distribution is defined as the most frequent value from a fitted distribution, which generally captures the highest likelihood of occurrence for a given variable. So, for each variable at every aspect ratio (i.e., for each unique distribution as described in the previous paragraph), an array of distributions are fit to a probability density 

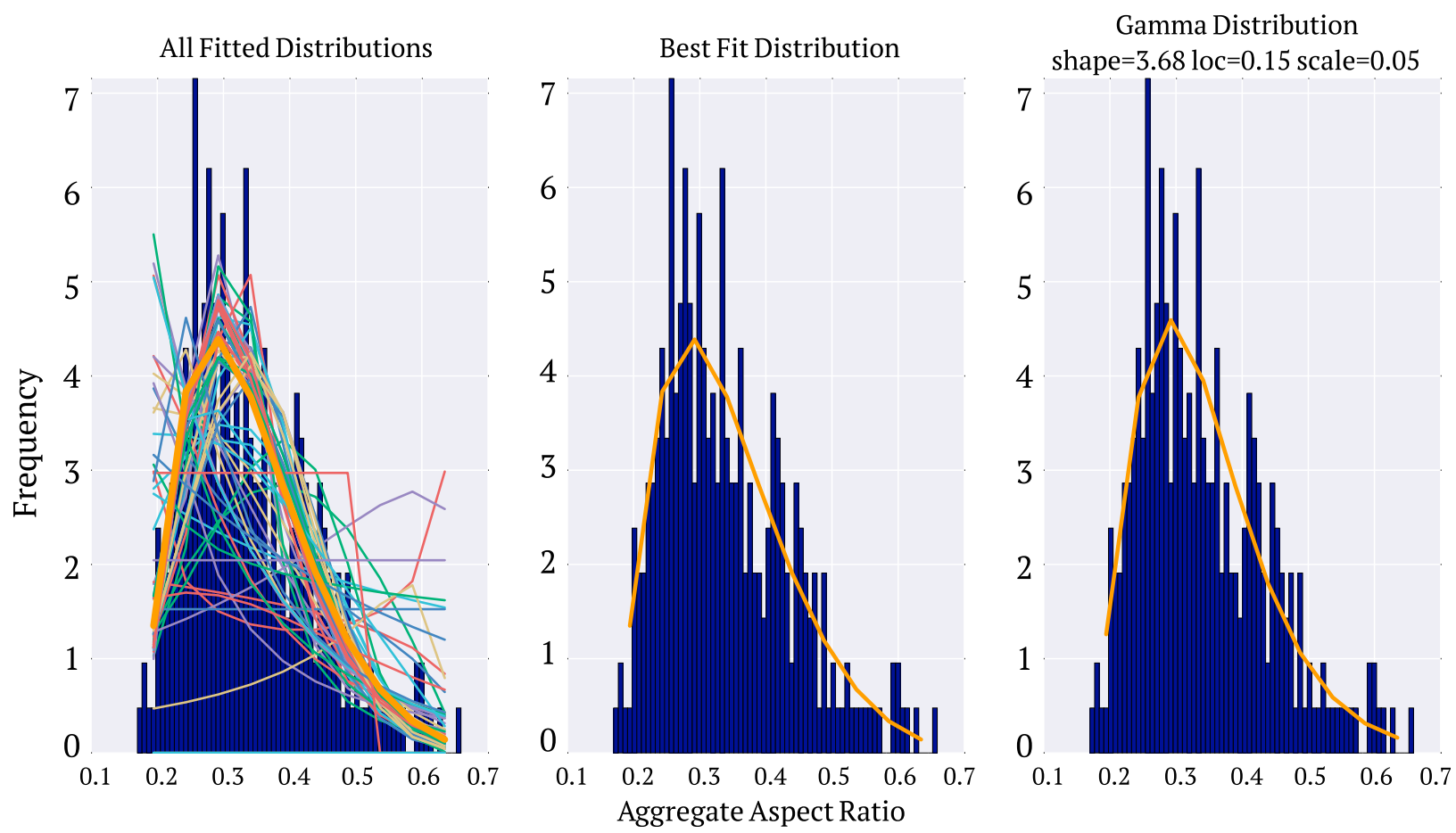

FIG. 6. Distribution analysis for 300 aggregates as an example from computed aggregate aspect ratio. (left) All fitted distributions, (center) the fitted distribution that minimizes the sum of square errors- "best distribution" (orange line), and (right) the fitted gamma distribution (orange line).

function of the data (Fig. 6, left). The distribution that best fits the data according to the least sum of square errors is determined, and the characteristic value of that fit, or the peak, is recorded (Fig. 6, center). This bulk characteristic value is used throughout the remainder of this paper to define resulting aggregate properties, and will be used in future work concerning aggregate values to be expected of ice-ice aggregation in bulk microphysical models. The fitted bulk peak is desired over the binned peak for this purpose (i.e., amenability to bulk modeling); moreover, the bulk peak considers not only the most frequent value, but also secondary peaks that add weight to the distribution. The binned peak would neglect these contributions.

Note that the fitted distributions used in the methodology outlined above do not always conform to the same function. For example, situations exist where a bimodal or multimodal distribution results, containing random peaks in the data that do not necessarily follow the overall trend of the bulk. Hence, it was chosen to fit the distribution to most accurately capture the peak frequency. By fitting the data using multiple distributions, a smoother trend is evident for plotting purposes, where, for example, a gamma distribution is not the best fit to reduce the sum of square errors. However, considering many bulk models use a gamma distribution to represent the data and that the gamma distribution encompasses other distributions, such as the exponential and lognormal, the fitted gamma distribution (Fig. 6, right) will be used when creating the lookup tables for bulk parameters. For all parameters studied thus far, the gamma distribution appropriately fits the data (no variable has negative skew) and can be easily implemented into bulk models.

\section{Results}

\section{a. Characteristic aggregate aspect ratio}

It is found that upon aggregation, the percent decrease (increase) of the aggregate aspect ratio for columns (plates) from the initial monomer aspect ratio is greater as habits become more extreme (Fig. 7). For example, a column that starts at $\phi=70.0$ aggregates to $\phi \sim 2$, whereas a column that starts at $\phi=5.00$ also aggregates to $\phi \sim 2$. This aligns with the observation that, generally, columns and plates become less extreme upon aggregation (Fig. 7), meaning that their aggregate aspect ratios move toward unity or a more isometric habit. Less extreme aggregate aspect ratios are predominantly attributable to high overlap and larger contact angles. Except in the rare cases that two monomers collect with 


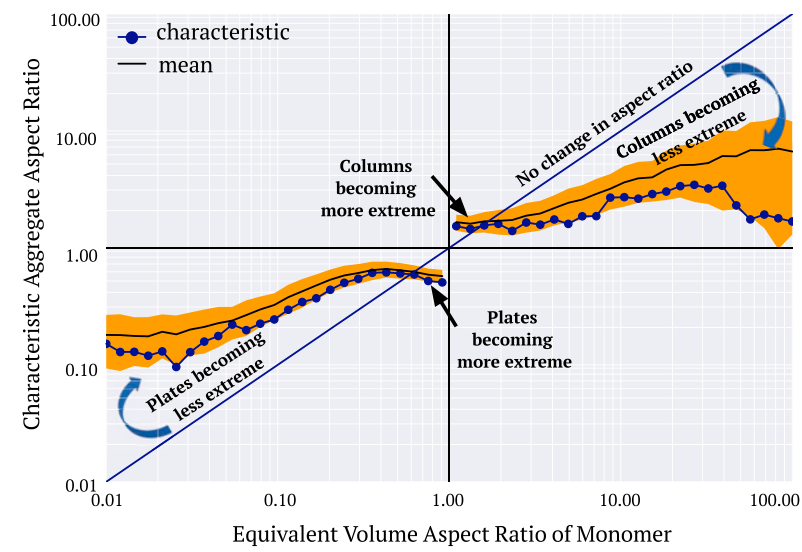

FIG. 7. Characteristic aggregate aspect ratio (blue dotted line) from the best-fit distribution (for smoothness in trend) with respect to monomer aspect ratio on the same scale for 300 aggregates each. Orange shading represents the mean (black line) plus and minus one standard deviation. The diagonal blue line from the bottom left to the upper right falls along equal $\phi$ values for both monomers and aggregates, indicating no change in $\phi$ with aggregation.

either $100 \%$ overlap or $0 \%$ overlap (i.e., "tip-to-tip"), the resulting aggregate major axis $L$ will always be between 1 and 2 times the length of each monomer $l$ (i.e., $l<L<2 l$ ). The major axis approaches the length of each monomer with increasing overlap. After aggregation, the depth of the aggregate at least doubles and is larger for larger contact angles.

Plates (columns) need to increase (decrease) their aspect ratio to become less extreme, meaning that the aggregate $c$ axis ( $a$ axis) must increase faster than the aggregate $a$ axis ( $c$ axis) with dependency on the initial magnitude of the monomer $a$ - and $c$-axis lengths. Normalization of the change in axis lengths corrects for the logarithmic nature of aspect ratio and its derivatives (i.e., plates $0 \leq \phi<1$; columns $\phi>1$ ). Figure 8 shows the change in aggregate major axis from monomer major axis and aggregate depth from monomer depth across all monomer aspect ratios. The minor axis (aggregate depth) increases at a larger rate than the majoraxis length, indicating the significance of contact angle. Interestingly, the change in both axis lengths for plates and columns is nearly identical after normalization despite randomization in overlap, contact angle, and variance in the 1D (2D) nature of the prism (basal) face for columns (plates) (Fig. 8).

As mentioned above, once two crystals collect, the depth (new minor-axis length of aggregate) will always double at a minimum with no contact angle (unless two columns aggregate parallel or quasi-parallel before reorientation), whereas the major axis does not double with any overlap (unless collecting tip-to-tip). Figure 9 further proves this point as the distance from the dashed

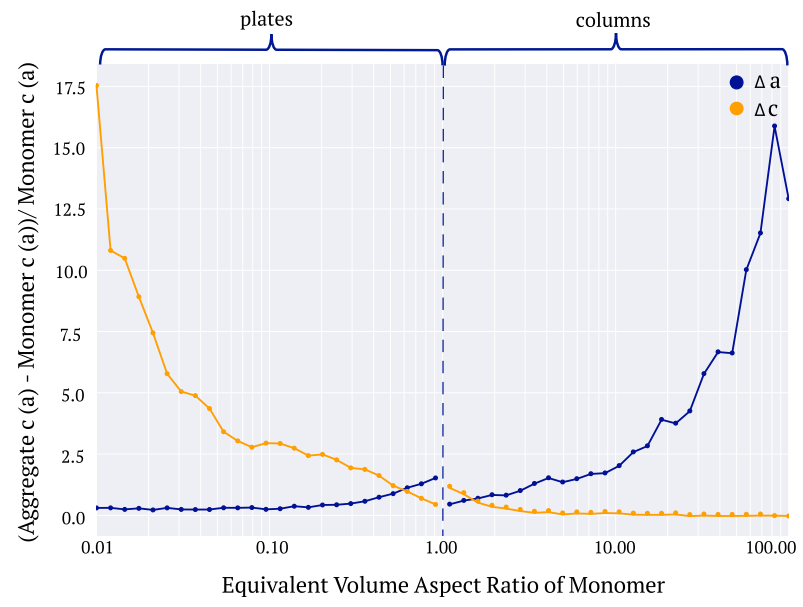

FIG. 8. Normalized change in the characteristic axis lengths from a gamma distribution by monomer $a$ - and $c$-axis lengths for 300 aggregates per monomer aspect ratio. The $a(c)$ axis is the major axis (depth) for planar particles. The $a(c)$ axis is the depth (major axis) for columnar particles.

orange lines (monomer depth) to the orange dotted line (characteristic aggregate depth) is significantly larger than the change in the major axis from the fitted ellipse (blue dashed to blue dotted). Consistently, the characteristic aggregate aspect ratios and axis lengths are lower than the mean due to positively skewed distributions, especially for extreme habits (Figs. 7 and 9). Moreover, for all aspect ratios, there is more spread (shaded area) in the depth measurement of the 300 aggregates than the major axis, concluding that the variability in the resultant aggregate aspect ratio is mostly due to varied contact angles (Fig. 9).

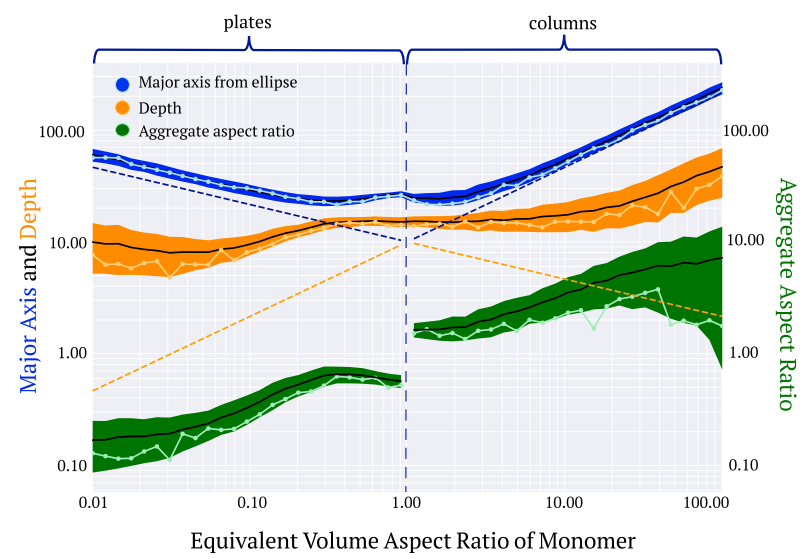

FIG. 9. Characteristic aggregate depth (orange), major axis (blue), and aspect ratio from the best distribution (for smoothness in trend). Monomer depth (dashed orange) and major axis (dashed blue) from an equivalent-volume sphere with a constant volume for all aspect ratios. Shading is plus and minus one standard deviation from the mean (solid black). 

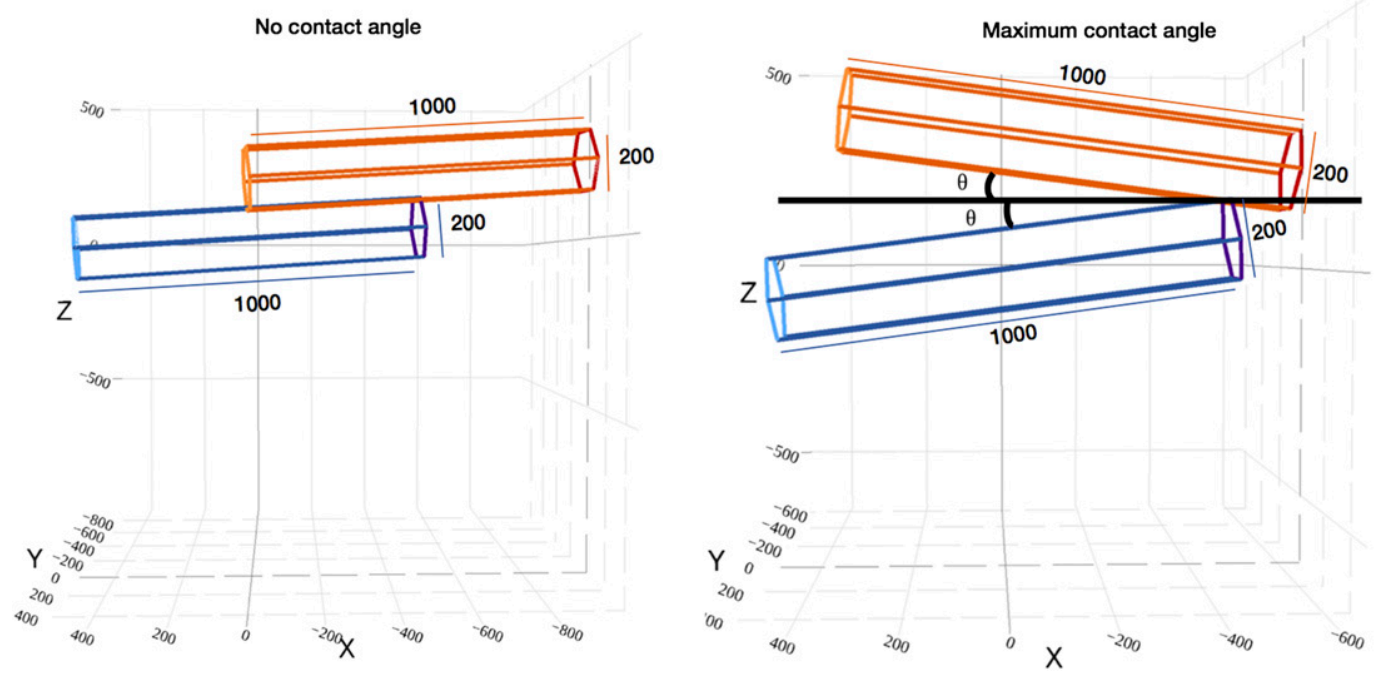

FIG. 10. Contact angle between monomers is determined by the amount of rotation around the $x$ and $y$ axes $\left(0^{\circ} \leq\right.$ contact angle $\leq 2 \theta$ ).

Initially, it was hypothesized that columns would become less extreme faster than plates upon aggregation because their major axis ( $c$ axis) can only be increased along one dimension, meaning there is less of a chance to maintain high columnar aggregate aspect ratios compared to planar aggregates where the major axis ( $a$ axis) can grow in both $x$ and $y$ for more extreme aspect ratios (low values). Results prove that plates and columns become less extreme at the same rate, which is of utmost importance in understanding the feedback of depositional growth for newly formed aggregates in habit-dependent aggregation models.

To perform the statistics in Figs. 7 and 9, 300 unique aggregates for each monomer aspect ratio were created, meaning that for each monomer aspect ratio, a dataset of 300 aggregate aspect ratios was created. From this distribution, a "characteristic" aggregate aspect ratio value was chosen, which was the most frequent (or the peak of the distribution). Because of this, there is no doubt that aggregate aspect ratios resulted that were less extreme than the characteristic (and there were many that were more extreme). While outliers certainly exist, on average, (or on characteristic in this case), and within one standard deviation, the resultant aggregate aspect ratio is always less extreme than the original monomer, except when the original monomers were quasi-spherical (aspect ratios close to unity). This result is a generalization due to the caveats listed in the discussion, but a relatively sound result within the bounds of IPAS and the approach used.

\section{b. Contact angle}

Contact angle was randomized between $0^{\circ}$ (where the crystals are completely flat on top of each other) and
2 times the maximum angle $\theta$ each crystal can have with the horizontal in both the $x$ and $y$ directions when crystal orientation is optimized (Fig. 1). To visualize the maximum possible contact angle, assume both crystals have been rotated around the $x$ and $y$ axes such that their projected area is optimized, with a greater tilt for thicker crystals. One crystal has no rotation around the $z$ axis and the other is rotated $180^{\circ}$ (one positive and one negative slope; Fig. 10). In this case, the contact angle is 2 times the angle that each crystal makes with the horizontal.

After reorientation for the maximum projected area, the maximum contact angle $\theta$ is no larger than $0.6^{\circ}$ for the longest columns, $66^{\circ}$ for "isometric hexagons" (quasi-spheres), and $2^{\circ}$ for the most extreme plates (Fig. 11). Remember that all particles are falling nearly flat in a perfectly vertical trajectory, disallowing for upright-oriented "T formation" aggregates. For comparison, Hashino and Tripoli (2011a) set the largest angle to be $45^{\circ}$ following Chen and Lamb (1994). Plates have twice the number of possible contact angles due to rotation around both the $x$ and $y$ axes compared to columns, which are only rotated around one horizontal axis. The larger angle in either the $x$ or the $y$ direction that results in the maximum projected area is used as the maximum contact angle for plates.

Because IPAS does not currently model intricate branches, the contact angle is near zero for two pristine plates (e.g., lack of interlocking dendritic arms), as is the case in Hashino and Tripoli (2011a). Moreover, Hashino and Tripoli (2011b) performed contact angle sensitivity tests in SHIPS by setting the angle between the aggregating crystals to $0^{\circ}$ and $90^{\circ}$. The larger angle resulted in 


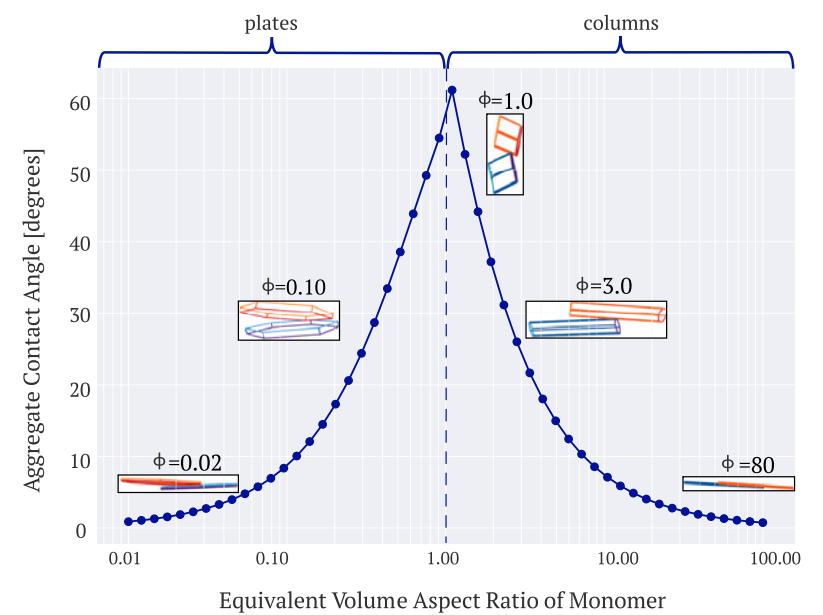

FIG. 11. Maximum possible contact angle between two aggregating crystals as a function of monomer aspect ratio. The embedded images show an example of an aggregate with its respective maximum contact angle.

smaller and denser aggregates, but the amount of testing from only the two angles limits comprehensive results. Aggregated needles were constrained to have no contact angle during the early stages of growth in SHIPS, similar to the small maximum contact angle values output from IPAS (Fig. 11). The ability to vary the contact angle within IPAS is an improvement over past models that specified one value and unrealistically bounded the distribution of aggregate aspect ratios. Within each 300-aggregate run, the IPAS distributions could potentially have 300 different contact angles. The ability to select the characteristic value from each distribution can improve current models that use this parameter.

\section{c. Overlap}

Variability in aggregate aspect ratio is additionally characterized by a broad range in the amount of overlap or separation between each monomer. The versatility of IPAS presents opportunities to test multiple methodologies to best represent overlap to provide insight into aggregate properties and for eventual translation to microphysical parameterizations. Two calculations are investigated herein, separation ratio $S$ and overlap percentage $O \%$.

\section{1) Separation RAtio}

A separation ratio was first developed by Higuchi (1960) and defined by the distance between the centers of the two particles $l$ and the diameters of each crystal $d_{1}$ and $d_{2}$-taken to be the longest distance between two points on the monomer:

$$
S=\frac{2 l}{d_{1}+d_{2}}
$$

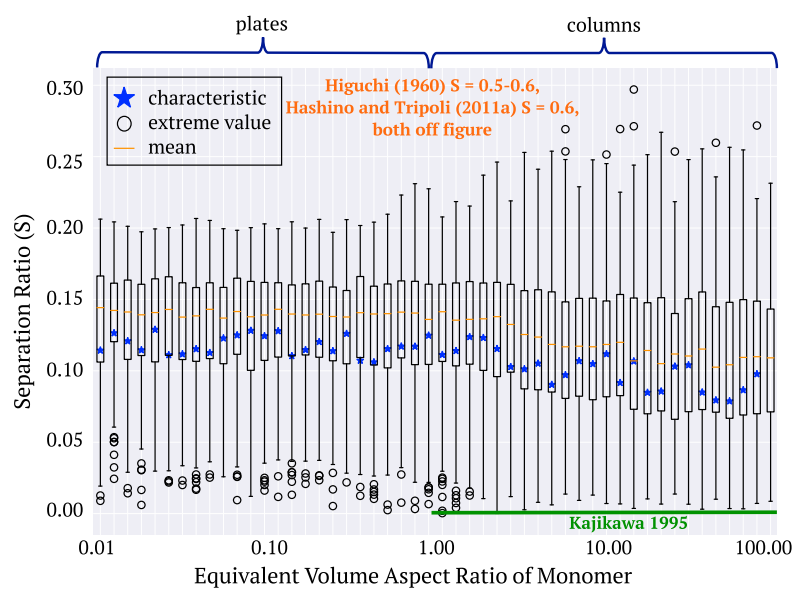

FIG. 12. Box-and-whisker plots of the separation ratio for 300 aggregates per monomer aspect ratio. The blue stars represent the characteristic or most frequent value of the fitted distribution, and the orange horizontal lines within the box indicate the mean. Boundaries of the box indicate the 25th (quartile 1, Q1) and 75th (quartile 3, Q3) percentiles, and the whiskers are drawn between $\mathrm{Q} 1$ - 1.5IQR and Q3 + 1.5IQR with the interquartile range (IQR) being Q3 - Q1. Outliers (circles) are defined outside of this range.

Here, $S$ is equal to 0 when the two crystals overlap completely at the centers and is equal to 1 when they are combined "tip-to-tip."

Following the prior studies of Higuchi (1960) and Hashino and Tripoli (2011a), the separation ratio is calculated in the overhead view ( $x-y$ plane) and used to determine how far apart the centers of the monomers are after aggregation. Note, $S$ is measured in IPAS before the aggregate is reoriented so that the orientation with the maximum projected area is selected (Fig. 2), although negligible variation in trend or magnitude was shown when calculated after aggregate reorientation.

From ground observations in cirrus clouds on Mount Tokachi, Higuchi (1960) found that the value of $S$ for two planar crystals or dendrites was most frequently reported to be about $0.5-0.6$. IPAS-calculated values of $S$ in Fig. 12 are considerably low compared to the previously mentioned observations. Although $S$ appears to have only a slight dependence on aspect ratio (particularly for columns), the assumed value of $S=0.6$ in Hashino and Tripoli (2011a) for planar crystals (and polycrystals) appears to be an overestimate. The benefit of $S$ in this study is the ability to confirm certainty in its magnitude for $n_{a}$ aggregates per aspect ratio.

Kajikawa (1995) observed that two similarly sized needle crystals tend to attach with their centers close $(S \sim 0)$ but with a $90^{\circ}$ angle between their $c$ axes (cross adhesion or high offset). While the centers of columnar aggregates are generally closer than planar aggregates due to less surface area to collect and farther intersection, 
$S$ does not regularly compute to be as low as 0 (Fig. 12). Owing to nearly unbounded randomization in collection overlap, meaning any distance between crystal centers is allowed as long as they intersect, $S$ naturally tends to higher values when $\phi>1$ using IPAS. Collection overlap is solely bounded by placement of the new crystal within a box that encompasses the seed crystal minimum and maximum $x$ and $y$ points. Enhancements to overlap constraints will be presented in future work.

It is noteworthy that Hashino and Tripoli (2011b) proved that the predicted bulk properties of the aggregates $(m-D$ relationships, mass-terminal velocity relationships, mean size, etc.) were largely dependent on the selected value of $S$ and secondarily on the contact angle. In contrast, Figs. 7 and 8 show that a change in aspect ratio toward less extreme values was most attributable to a larger change in the depth of the aggregate relative to the major-axis length, suggesting a stronger dependency on contact angle relative to separation ratio, which is related to contact angle, not overlap.

\section{2) OVERLAP PERCENTAGE}

While the separation ratio calculation proposed by Hashino and Tripoli (2011a) described above provides some insight into overlap, $S$ is less informative for extreme columns due to the high likelihood of some offset from random rotation around the $z$ axis, (i.e., " $\mathrm{X}$ " formation). Hence, a secondary overlap parameter was developed for IPAS that relates the intersected area between the seed crystal and falling crystal relative to the total area of both crystals in the top-down perspective or $x-y$ plane:

$$
O_{\%}=\frac{A_{i j}}{A_{i}} \times 100
$$

where $O_{\%}$ is the percentage of overlap, $A_{i}$ is the area of the seed crystal, and $A_{i j}$ is the area of overlap (see Fig. 3 for illustration).

Figure 13 relates IPAS-calculated $O_{\%}$ and distribution characteristics to monomer aspect ratio for 300 aggregates per aspect ratio. The characteristic value of the distribution is always considerably lower than the mean, attributable to right-skewed distributions. The outliers in Fig. 13 are not "bad data" but represent the unlikelihood, for example, of two long columns to overlap with nearly parallel edges (minimal offset) or two wide plates to overlap nearly on top of each other. While it is possible to achieve $O_{\%}=100$, the probability of this occurrence is small due to complete randomness in offset and overlap for each of the 300 aggregates. Of course, there is a better probability of $O_{\%} \simeq 100$ should more

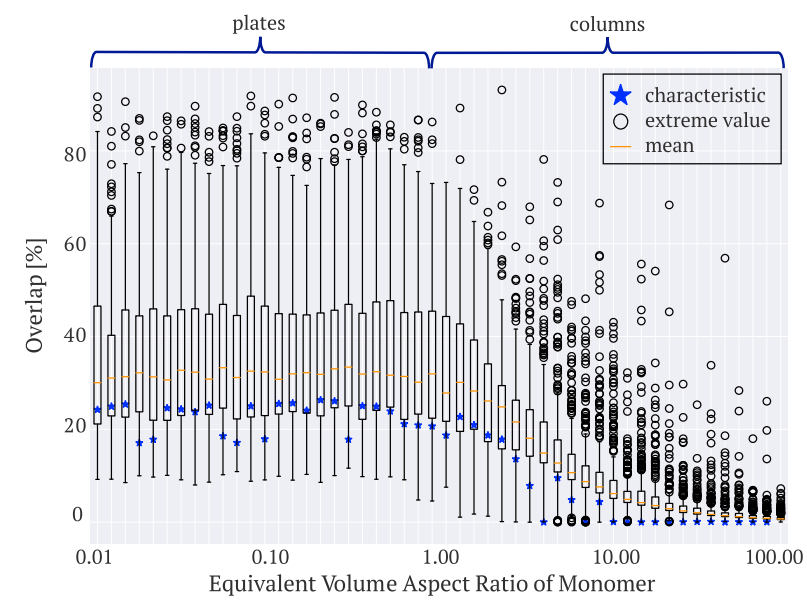

FIG. 13. Box-and-whisker plots of the overlap percentage for 300 aggregates per monomer aspect ratio. The blue stars represent the characteristic or most frequent value of the fitted distribution, and the orange horizontal lines within the box indicate the mean. Boundaries of the box indicate the 25th (Q1) and 75th (Q3) percentiles, and the whiskers are drawn between Q1 - 1.5IQR and Q3 + 1.5IQR with IQR being Q3 - Q1. Outliers (circles) are defined outside of this range.

aggregates be formed, but this study is concerned with capturing the most likely value; thus, a completely filled distribution with $O$ \% from 0 to 100 is not necessary.

Plates consistently have high $O_{\%}$ values and are well dispersed compared to columns that are positively skewed with almost all aggregates minimally overlapping to form various degrees of cross adhesion or X's (Fig. 13). The wide, 2D basal faces that collect for plates provide higher $O \%$ values compared to the relatively long, narrow prism faces of columns. Since columns tend to collect in an $\mathrm{X}$ formation, there is a minimum $O_{\%}$ value proportional to the width of the prism face but always above $0 \%$. For this reason, the outliers that produce small $O \%$ values for columns suggest that the edge of the new crystal only partially aggregated on the prism face of the seed crystal (i.e., to form a " $T$ " instead of an "X").

With overlap computed as an area percentage, even though columns tend to aggregate with their centers close, $O \%$ is decreased appropriately as the $c$ axes rotate away from each other. Hashino and Tripoli (2011a) make up for this by forcing the $S$ parameter and contact angle to zero for columnar aggregates made up of any monomer with $\phi>5$. Overlap as an area percentage in IPAS naturally decreases to zero near this value.

The ability to evolve $O \%$ with aspect ratio in IPAS may shed light on particle size distribution (PSD) evolution in microphysical parameterizations. High (low) overlap leads to a decreased (increased) majoraxis length, higher (lower) aggregate density, and faster (slower) terminal velocities. Changes in the major-axis 
length of a particle not only shifts the PSD due to growth by collection, but results in a reduction in aspect ratio (i.e., the loss of two-monomer crystals with extreme aspect ratios to one aggregate with a less extreme aspect ratio), which directly affects the rate at which the crystals grow via vapor deposition for a particular bulk distribution. Further, the increase in mass and change in density control largely the sedimentation and fallout rates, further impacting the rate of evolution of the PSD. Furthermore, it is expected that $S$ should itself evolve as more particles collide and coalesce into a larger aggregate, something that will be examined but is outside the scope of two-monomer collection thus far.

\section{Discussion and conclusions}

Hydrometeors composed of ice are complex and nonuniform in shape, especially with regard to density differences among crystals of similar mass; however, predefined categories that neglect smooth particle transitions are neither theoretically accurate nor practical due to the number of prognostic variables that would be needed to accurately depict the multitude of habits evident in cold clouds. Ice microphysical processes in mixed-phase clouds need to be accurately represented to properly evolve PSDs through changing habit characteristics, and thus fall speed variations, all of which alter aggregation rates both at cloud level and at the surface. Contrary to prior models that limit aggregation to particular categorized habits (dendrites, needles, columns, etc.) (Woods et al. 2007; Thompson et al. 2008; Hashino and Tripoli 2011b), bulk models such as the adaptive habit model (AHM) and $\mathrm{P} 3$ prognostically evolve ice particle characteristics without dependence on equivalent volume spheres or mass-dimensional relationships. However, to appropriately parameterize microphysical properties in these advanced bulk-evolving schemes, accurate hydrometeor properties and bounds are required. Although some laboratory data exist to meet these needs (e.g., Hosler et al. 1957; Fukuta and Takahashi 1999; Bailey and Hallett 2009; Heymsfield and Westbrook 2010; Connolly et al. 2012), gaps remain due to the inherent inability for laboratory experiments to generate large amounts of data on which to perform statistical analysis.

The offline theoretical laboratory of IPAS is an innovative approach in simulating aggregation and in understanding the influence that monomer properties have on aggregate characteristics. Through the use of IPAS, the collection process is more precisely depicted, is designed with bulk modeling in mind, and is efficiently updated in a resource-conscious sense. Similar to using laboratory data to apply bounds to the amount of variability for each parameter discussed (e.g., aspect ratio, overlap, contact angle), IPAS is used as the "laboratory" to provide the necessary statistical and visual framework for depicting distributions in bulk models.

Joining IPAS-derived aggregate properties with bulk model time evolution capabilities facilitates the ability to more accurately evolve PSDs through aggregate dimensions that vary as a function of aspect ratio and crystal habit. IPAS has been designed so that bulk models will predominantly use IPAS-determined $a_{a}$ and $c_{a}$-axis lengths to calculate aggregate aspect ratios after collection. The methodology to determine the axis lengths is primarily a function of contact angle and overlap, two parameters that have been updated via random functions and distribution analysis to justify the idea that no two aggregates will obtain the same values upon collection. Still, a reasonable range for the aggregate major-axis length, depth, overlap, separation, maximum contact angle, and aspect ratio is determined.

The versatility of IPAS allows for the capability to create enough aggregates (according to given monomer aspect ratio and size) to generate a robust distribution for many different initial conditions (e.g., monomer attributes). Moreover, multiple distributions are created for many aggregate properties (e.g., aggregate aspect ratio, $a_{a}, c_{a}$, change in density) Once a distribution is created, bulk properties are then extracted from that distribution, including characteristic and mean values. Lookup tables containing those bulk properties are then generated, again as a function of monomer properties. Because these monomer properties are predicted and evolve in microphysics models (the AHM in this case), we are able to attribute bulk properties to the aggregates that are created in the model using the lookup table. More details on the integration and outcome of these data and application in the AHM will be the topic of future work.

Initial investigations using IPAS are presented herein. First, aggregation of two plates or columns produces aspect ratios that tend toward an isometric shape and is moderated faster for more extreme habits (Fig. 7). This was primarily due to varied contact angles that increase the depth from the side view faster than the increase in the major-axis length. It was unanticipated that the change in the major-axis length and depth measurement from monomer to aggregate was nearly identical given randomization in each parameter constrained by different bounds. It is expected that this result will be crucial in accurately predicting sedimentation rates at cloud base and snowfall accumulation near the surface from altered depositional growth rates and the conversion from ice to aggregates.

The distance between the centers of the monomers and amount of overlapped area is another important 
piece in accurately determining the collection efficiency of crystals. Columns tend toward low area overlap percentages but have close centers compared to plates which have higher area overlap percentages and centers that vary in proximity (Figs. 12 and 13). In more detailed models, it is expected that the separation and overlap of monomers will influence PSDs and aggregate density via mass changes from depositional growth.

While the other aggregate properties that are presented in the results are not typically found in bulk models (e.g., overlap and contact angle), they are presented herein in order to convey the level of detail provided by IPAS. This detail is valuable in developing an intricate understanding of the aggregation process in IPAS, allows for a level of control by the modeler in extracting information during the entire aggregation process, and illustrates potential parameters that may be useful for comparisons to particle imagery (e.g., CPI data) in future work.

A newly formed method to vary the contact angle between two crystals has built upon prior studies that fixed the collection of crystals solely to $0^{\circ}, 45^{\circ}$, or $90^{\circ}$ for simple geometry (Hashino and Tripoli 2011b), which did not allow for variability in collection orientation. At this time, the contact angle between two monomers is constrained by the falling orientation that produces the maximum projected area, with some tilt expected (Fig. 1), which allows for a range of contact angles and depth measurements. Isometric crystals have the largest maximum contact angle, whereas extreme crystals, defined by aspect ratio, have virtually no contact angle due to their thin nature (Fig. 11). A standardized yet modifiable approach to the falling orientation of both the monomers and the aggregate has been implemented and can be used in future studies to potentially add turbulent effects, interlocking branches, etc.

Hashino and Tripoli (2011a) suggest that crystals with more complicated shapes, lower densities, and larger sizes can aggregate with larger angles upon collection. While the results presented in Fig. 11 are the inverse of this assumption, these initial IPAS simulations contain the following caveats:

1) As in many modeling studies, the monomers are always assumed to fall with their maximum dimension perpendicular to the flow, and based on the explanation provided above of the effect of overhang, this assumption implies a direct relationship between monomer aspect ratio and contact angle with the horizontal plane (and thus the secondary monomer). Offline investigations of completely random falling orientation (i.e., no constraints based on projected area) result in no discernible trend in contact angle with respect to aspect ratio.
2) The monomers presented thus far are perfect hexagonal prisms and cover most possibilities of planar aggregation. However, in reality, as aspect ratio deviates further from unity, the planar ice crystals will begin to develop intricate branches or dendritic arms, which would allow for intersecting collection and alter the contact angle (among other parameters). Ice crystals in nature also extend well beyond pristine plates and columns; next steps with IPAS include representing more complex crystals, such as dendrites and bullet rosettes, with a trade-off for increased necessary computational power for larger polygonal point arrays.

3) IPAS simulations thus far aggregate two identical crystals, which allows for the constraint of contact angle from $0^{\circ}$ to $2 \theta$ (Fig. 10). With attempts to extend IPAS-extracted parameters to bulk microphysical models, this final constraint is appropriate for predicting the characteristic value of a distribution within a grid box. However, work is underway to aggregate monomers of differing aspect ratios, altering this flowdefined constraint to range from $0^{\circ}$ to $\theta_{1}+\theta_{2}$.

Despite identical starting characteristics currently in IPAS, there is heterogeneity in ice crystal growth in nature within a temperature regime due to intricacies in vapor deposition. Even though crystals can have the same mass, differing habits lead to varied fall speeds unlike with water droplet growth (Connolly et al. 2012). While the conclusions presented are verified for two identical crystals, work is underway to broaden the findings for multiple, nonidentical crystals, all originating from the same perceived distribution, that encompass more intricacies as in nature. Such implementations to improve precision in aggregation models are arduous yet important due to the multitude of systems in which aggregation takes place.

It is known that aggregation alters depositional growth rates-this research highlights the dependence of monomer properties to aggregate growth through a consistent methodology that determines the aspect ratio of the formed aggregate. Subsequently, fall speeds, particle mass and size distributions, and cloud cover/radiation structure in mixed-phase clouds can be improved and more accurately evolved, which is already acknowledged as important by Locatelli and Hobbs (1974), Fukuta and Takahashi (1999), and Lamb and Verlinde (2011).

The purpose of this theoretical study is to demonstrate IPAS capabilities prior to a complete verification phase of simulated and observed ice crystals and aggregates that is outside the scope of this study. A preliminary methodology has been defined to verify the idealized nature of IPAS with CPI data [similar to the technique used by Schmitt and Heymsfield (2014) but for aggregates 
that are composed of a few monomers]. Using IPAS, a large dataset of two-monomer aggregates will be generated with a random orientation to mimic the turbulent environment inside the CPI probe. IPAS-computed aspect ratio, area ratio, and complexity (Schmitt and Heymsfield 2014) will be compared to likely subsets of CPI data for similar particle attributes. To ensure consistency in verification with CPI images, IPAS properties need to be identically calculated to CPI imagery, meaning a $2 \mathrm{D}$ aspect ratio calculation will be invoked on IPAS particles. While there is no perfect method to compare 3D IPAS-calculated aspect ratios to aspect ratios computed on 2D CPI images, the inclusion of multiple IPAS-generated parameters will further authenticate IPAS. Additionally, IPAS has the ability to represent different habit types beyond the plates and columns presented here (i.e., dendrites and bullet rosettes). The additional habits can provide supplementary validation across many particle types.

Acknowledgments. V. Przybylo, K. Sulia, C. Schmitt, and Z. Lebo would like to thank the Department of Energy for support under DOE Grant DE-SC0016354. K. Sulia is additionally supported through an appointment under the SUNY 2020 Initiative. W. May is supported by the Center of Excellence in Weather Enterprise. The authors would also like to thank collaborators Drs. Jerry Harrington and Hugh Morrison as well as the ASRC Extreme Collaboration, Innovation, and Technology (xCITE) Laboratory for IPAS development support. An early version of IPAS has been made open source (https://github.com/ASRCsoft/IPAS_research; Przybylo et al. 2019). The National Center for Atmospheric Research (C. Schmitt) is sponsored by the National Science Foundation.

\section{REFERENCES}

Bailey, M. P., and J. Hallett, 2009: A comprehensive habit diagram for atmospheric ice crystals: Confirmation from the laboratory, AIRS II, and other field studies. J. Atmos. Sci., 66, 28882899, https://doi.org/10.1175/2009JAS2883.1.

Biggerstaff, M. I., and R. A. Houze Jr., 1991: Kinematic and precipitation structure of the 10-11 June 1985 squall line. J. Atmos. Sci., 119, 3034-3065, https://doi.org/10.1175/1520-0493(1991) 119<3034:KAPSOT>2.0.CO;2.

Chen, J.-P., and D. Lamb, 1994: The theoretical basis for the parameterization of ice crystal habits: Growth by vapor deposition. J. Atmos. Sci., 51, 1206-1222, https://doi.org/10.1175/ 1520-0469(1994)051<1206:TTBFTP>2.0.CO;2.

Churchill, D. D., and R. A. Houze Jr., 1984: Development and structure of winter monsoon cloud clusters on 10 December 1978. J. Atmos. Sci., 41, 933-960, https://doi.org/10.1175/ 1520-0469(1984)041<0933:DASOWM >2.0.CO;2.

Connolly, P. J., C. Saunders, M. Gallagher, K. Bower, M. Flynn, T. Choularton, J. Whiteway, and R. Lawson, 2005: Aircraft observations of the influence of electric fields on the aggregation of ice crystals. Quart. J. Roy. Meteor. Soc., 131, 16951712, https://doi.org/10.1256/qj.03.217.

— , C. Emersic, and P. R. Field, 2012: A laboratory investigation into the aggregation efficiency of small ice crystals. Atmos. Chem. Phys., 12, 2055-2076, https://doi.org/10.5194/ acp-12-2055-2012.

Cotton, W. R., G. J. Tripoli, R. M. Rauber, and E. A. Mulvihill, 1986: Numerical simulation of the effects of varying ice crystal nucleation rates and aggregation processes on orographic snowfall. J. Atmos. Sci., 25, 1658-1680, https://doi.org/10.1175/ 1520-0450(1986)025<1658:NSOTEO > 2.0.CO;2.

Cox, G. P., 1988: Modeling precipitation in frontal rainbands. Quart. J. Roy. Meteor. Soc., 114, 115-127, https://doi.org/ 10.1002/qj.49711447906.

Fanning, D. W., 2002: The Coyote library. GitHub, http:// www.idlcoyote.com/programs/fit_ellipse.pro.

Fukuta, N., and T. Takahashi, 1999: The growth of atmospheric ice crystals: A summary of findings in vertical supercooled cloud tunnel studies. J. Atmos. Sci., 56, 1963-1978, https://doi.org/ 10.1175/1520-0469(1999)056<1963:TGOAIC >2.0.CO;2.

Gilmore, M., J. M. Straka, and E. N. Rasmussen, 2004: Precipitation uncertainty due to variations in precipitation particle parameters within a simple microphysics scheme. Mon. Wea. Rev., 132, 2610-2627, https://doi.org/10.1175/ MWR2810.1.

Harrington, J. Y., K. J. Sulia, and H. Morrison, 2013: A method for adaptive habit prediction in bulk microphysical models. Part II: Parcel model corroboration. J. Atmos. Sci., 70, 365-376, https://doi.org/10.1175/JAS-D-12-0152.1.

Hashino, T., and G. J. Tripoli, 2011a: The Spectral Ice Habit Prediction System (SHIPS). Part III: Description of the ice particle model and the habit-dependent aggregation model. J. Atmos. Sci., 68, 1125-1141, https://doi.org/10.1175/2011JAS3666.1.

_, and _ 2011b: The Spectral Ice Habit Prediction System (SHIPS). Part IV: Box model simulations of the habit-dependent aggregation process. J. Atmos. Sci., 68, 1142-1161, https:// doi.org/10.1175/2011JAS3667.1.

Heymsfield, A. J., and C. D. Westbrook, 2010: Advances in the estimation of ice particle fall speeds using laboratory and field measurements. J. Atmos. Sci., 67, 2469-2482, https://doi.org/ 10.1175/2010JAS3379.1.

Higuchi, K., 1960: On the coalescence between plane snow crystals. J. Atmos. Sci., 17, 239-243, https://doi.org/10.1175/1520-0469(1960) 017<0239:OTCBPS $>2.0 . C O ; 2$.

Hobbs, P. V., and W. D. Scott, 1965: A theoretical study of the variation of ice crystal habits with temperature. J. Geol. Res., 70, 5025-5034, https://doi.org/10.1029/JZ070i020p05025.

Hosler, C. L., and R. E. Hallgren, 1960: The aggregation of small ice crystals. Discuss. Faraday Soc., 30, 200-207, https://doi.org/ 10.1039/df9603000200.

_ D. C. Jensen, and L. Goldshlak, 1957: On the aggregation of ice crystals to form snow. J. Meteor., 14, 415-420, https://doi.org/ 10.1175/1520-0469(1957)014<0415:OTAOIC >2.0.CO;2.

Jorgensen, D. P., 1984: Mesoscale and convective-scale characteristics of mature hurricanes. Part I: General observations by research aircraft. J. Atmos. Sci., 41, 1268-1285, https://doi.org/ 10.1175/1520-0469(1984)041<1268:MACSCO > 2.0.CO;2.

Kajikawa, M., 1995: Characteristics of the aggregation of needle snow crystals. Seppyo, 57, 349-355.

, and A. J. Heymsfield, 1989: Aggregation of ice crystals in cirrus. J. Atmos. Sci., 46, 3108-3121, https://doi.org/10.1175/ 1520-0469(1989)046<3108:AOICIC>2.0.CO;2. 
Korolev, A., 2007: Limitations of the Wegener-Bergeron-Findeisen mechanism in the evolution of mixed-phase clouds. J. Atmos. Sci., 64, 3372-3375, https://doi.org/10.1175/JAS4035.1.

— clouds. Quart. J. Roy. Meteor. Soc., 129, 19-38, https://doi.org/ 10.1256/qj.01.203.

Kuroda, T., and R. Lacmann, 1982: Growth kinetics of ice from the vapour phase and its growth forms. J. Cryst. Growth, 56, 189205, https://doi.org/10.1016/0022-0248(82)90028-8.

Lamb, D., and P. V. Hobbs, 1971: Growth rates and habits of ice crystals grown from the vapor phase. J. Atmos. Sci., 28, 15061509, https://doi.org/10.1175/1520-0469(1971)028<1507: GRAHOI $>2.0 . \mathrm{CO} ; 2$.

_ Cambridge University Press, $600 \mathrm{pp}$.

Lin, Y. L., R. Farley, and H. D. Orville, 1983: Bulk parameterization of the snow field in a cloud model. J. Climate Appl. Meteor., 22, 1065-1092, https://doi.org/10.1175/1520-0450(1983)022<1065: BPOTSF $>2.0 . \mathrm{CO} ; 2$.

Locatelli, J., and P. V. Hobbs, 1974: Fall speeds and masses of solid precipitation particles. J. Geophys. Res., 79, 2185-2197, https:// doi.org/10.1029/JC079i015p02185.

Milbrandt, J. A., and H. Morrison, 2013: Prediction of graupel density in a bulk microphysics scheme. J. Atmos. Sci., 70, 410 429, https://doi.org/10.1175/JAS-D-12-0204.1.

Morrison, H., and J. A. Milbrandt, 2015: Parameterization of cloud microphysics based on the prediction of bulk ice particle properties. Part I: Scheme description and idealized tests. J. Atmos. Sci., 72, 287-311, https://doi.org/10.1175/JAS-D-14-0065.1.

, - _ , G. H. Bryan, S. A. Tessendorf, G. Thompson, and K. Ikeda, 2015: Parameterization of cloud microphysics based on the prediction of bulk ice particle properties. Part II: Case study comparisons with observations and other schemes. J. Atmos. Sci., 72, 312-339, https://doi.org/10.1175/JAS-D-14-0066.1.

Passarelli, R. E., 1978: Theoretical and observational study of snowsize spectra and snowflake aggregation efficiencies. J. Atmos. Sci., 35, 882-889, https://doi.org/10.1175/1520-0469(1978)035<0882: TAOSOS $>2.0 . \mathrm{CO} ; 2$.

Phillips, V. T. J., M. Formenton, A. Bansemer, I. Kudzotsa, and B. Lienert, 2015: A parameterization of sticking efficiency for collisions of snow and graupel with ice crystals: Theory and comparison with observations. J. Atmos. Sci., 72, 4885-4902, https://doi.org/10.1175/JAS-D-14-0096.1.

Pruppacher, H. R., and J. D. Klett, 1997: Microphysics of Clouds and Precipitation. Kluwer Academic, 954 pp.

Przybylo, V., C. Schmitt, W. May, K. Sulia, and Z. Lebo, 2019: The Ice Particle and Aggregate Simulator (IPAS). GitHub, https:// github.com/ASRCsoft/IPAS_research.
Reinhardt, T. U., and U. Wacker, 2004: Impact of ice particle habits on simulated clouds. Geophys. Res. Lett., 31, L21106, https:// doi.org/10.1029/2004GL021134.

Reisner, J. R., and R. T. Rasmussen, 1998: Explicit forecasting of supercooled liquid water in winter storms using the MM5 mesoscale model. Quart. J. Roy. Meteor. Soc., 124, 1071-1107, https://doi.org/10.1002/qj.49712454804.

Rogers, R. R., and M. K. Yau, 1989: A Short Course in Cloud Physics. Pergamon Press, 206 pp.

Rutledge, S. A., and R. A. Houze Jr., 1987: A diagnostic modeling study of the trailing stratiform region of a midlatitude squall line. J. Atmos. Sci., 44, 2640-2656, https://doi.org/10.1175/ 1520-0469(1987)044<2640:ADMSOT>2.0.CO;2.

Schmitt, C. G., and A. J. Heymsfield, 2010: The dimensional characteristics of ice crystal aggregates from fractal geometry. J. Atmos. Sci., 67, 1605-1616, https://doi.org/10.1175/ 2009JAS3187.1.

—- and ——, 2014: Observational quantification of the separation of simple and complex atmospheric ice particles. Geophys. Res. Lett., 41, 1301-1307, https://doi.org/10.1002/ 2013 GL058781.

,-- , P. Connolly, E. Jarvinen, and M. Schnaiter, 2016: A global view of atmospheric ice particle complexity. Geophys. Res. Lett., 43, 11 913-11 920, https://doi.org/10.1002/2016GL071267.

Sulia, K. J., and J. Y. Harrington, 2011: Ice aspect ratio influences on mixed-phase clouds: Impacts on phase partitioning in parcel models. J. Geophys. Res., 116, D21309, https://doi.org/ 10.1029/2011JD016298.

—_ - _ , and H. Morrison, 2013: A method for adaptive habit prediction in bulk microphysical models. Part III: Applications and studies within a two-dimensional kinematic model. J. Atmos. Sci., 70, 3302-3320, https://doi.org/10.1175/ JAS-D-12-0316.1.

Thériault, M. J., R. Rasmussen, K. Ikeda, and S. Landolt, 2012: Dependence of snow gauge collection efficiency on snowflake characteristics. J. Appl. Meteor. Climatol., 51, 745-762, https:// doi.org/10.1175/JAMC-D-11-0116.1.

Thompson, G., P. Field, R. Rasmussen, and W. Hall, 2008: Explicit forecasts of winter precipitation using an improved bulk microphysics scheme. Part II: Implementation of a new snow parameterization. Mon. Wea. Rev., 136, 5095-5115, https:// doi.org/10.1175/2008MWR2387.1.

Woods, C., M. Stoelinga, and J. Locatelli, 2007: The IMPROVE-1 storm of 1-2 February 2001. Part III: Sensitivity of a mesoscale model simulation to the representation of snow particle types and testing of a bulk microphysical scheme with snow habit prediction. J. Atmos. Sci., 64, 3927-3948, https://doi.org/ 10.1175/2007JAS2239.1. 